\title{
Effects of magnetic field on the runaway instability of relativistic accretion tori near a rotating black hole
}

\author{
V. Karas and J. Hamerský \\ Astronomical Institute, Academy of Sciences, Prague, Czech Republic \\ email: vladimir.karas@cuni.cz
}

\begin{abstract}
Runaway instability operates in accretion tori around black holes, where it affects systems close to the critical (cusp overflowing) configuration. The runaway effect depends on the radial profile $l(R)$ of the angular momentum distribution of the fluid, on the dimension-less spin a of the central black hole $(|a| \leqslant 1)$, and other factors, such as self-gravity. Here we discuss the role of runaway instability within a framework of an axially symmetric model of perfect fluid endowed with a purely toroidal magnetic field.
\end{abstract}

Keywords. Accretion, accretion disks - gravitation - black hole physics

\section{Introduction}

Relativistic accretion of material over the cusp transfers the mass and angular momentum onto the black hole, thereby changing the intrinsic parameters of the Kerr metric (Abramowicz et al. 1998, Rezzolla et al. 2003, Korobkin et al. 2013). Previously it was demonstrated that for the power-law dependence of the radial angular momentum profile, $l(R) \propto R^{q}$, non-magnetized tori always become runaway stable for a sufficiently high positive value of $q$ (Karas et al. 2004). Here we explore effects of the plasma $\beta$-parameter (the ratio of gas to magnetic pressure) and other parameters of the model on the evolution of critical configurations that happen to be just on the verge of cusp overflow.

The magnetic field causes small departures from the corresponding non-magnetic configuration in the early phases of accretion. We show that the toroidal magnetic component inside an accretion torus does not change the frequency of its oscillations significantly. We identify these oscillations as the radial epicyclic mode. These weak effects can trigger the runaway instability even in situations when the purely hydrodynamical regime of the torus is stable. We discuss examples of the torus evolution depending on the initial magnetization $\beta$, the slope $q$, and the spin $a$ (Kerr metric dimensionless parameter).

Within the framework of an axially symmetric magnetized fluid torus model we have extended the previous results on the onset of runaway instability of relativistic configurations near a rotating black hole. Based on our recent paper (Hamerský \& Karas 2013), these calculations are relevant for understanding the accretion process in the inner regions of accretion flows. We employ the critical (overflowing) solution as an initial configuration, which we perturb and evolve by using a $2 \mathrm{D}$ numerical scheme.

\section{Results}

The mass accretion can either bring the system out of the critical configuration and stabilize it with an updated set of model parameters and a modified profile of angular momentum, or the accretion process continues in a runaway mode and leads to a complete 

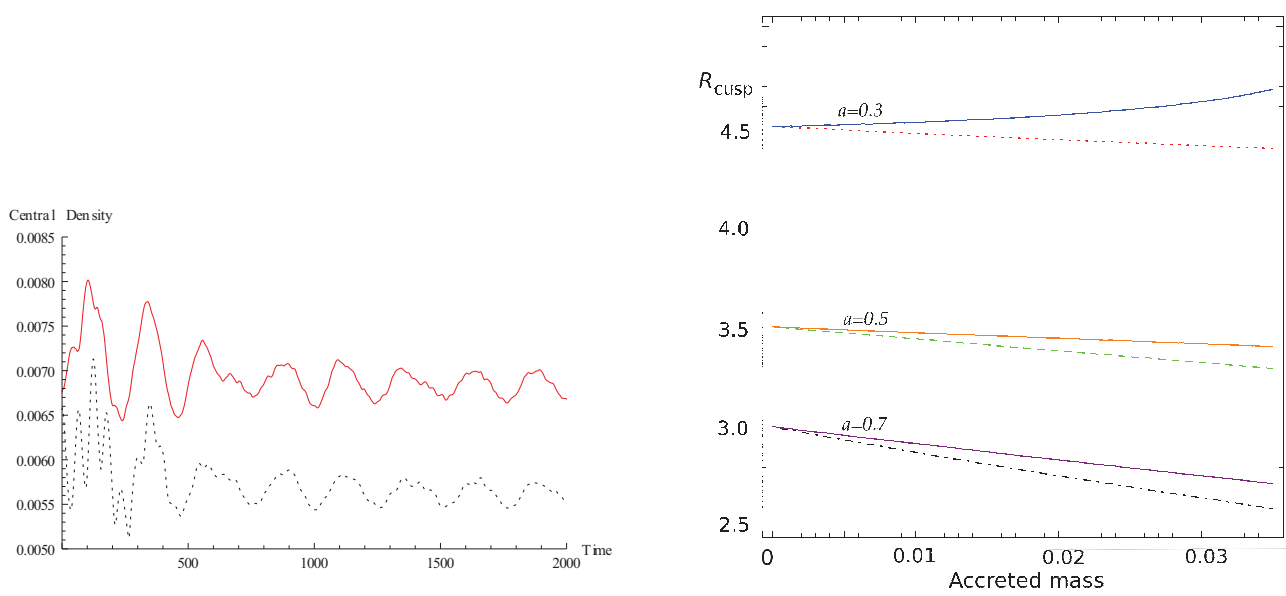

Figure 1. The changing torus structure during the accretion process. Left: evolving density of the perturbed configuration where the inner edge is pushed slightly outward, setting the torus out of its initial steady state. Oscillations of the central density are shown for the magnetized (dotted) vs. non-magnetized (solid line) cases. The magnetization parameter was set $\beta=3$. Right: the position of the inner cusp $R_{\text {cusp }}$ changing with the accreted mass (relative to the black hole mass) for different values of the initial spin $a=0.3,0.5$, and 0.7 . Broken/solid lines correspond to Keplerian/sub-Keplerian angular momentum density profile within the torus. [A COLOR VERsion is AVAILAble OnLINE.]

destruction of the torus. We concentrated on systems with radially increasing angular momentum density that are threaded by a purely toroidal magnetic field. We neglected self-gravity of the gaseous material (the mass of the torus was set to be at most several percent of the black-hole mass), nevertheless, we allowed for a gradual change of the Kerr metric mass and spin parameters by accretion over the inner edge. The angular momentum distribution within the torus was also allowed to evolve, starting from the initial power-law profile. The mass transfer influences the location of the cusp of the critical configuration, which can lead to the runaway instability.

If the profile of the angular momentum increases fast enough with the radius (typically, $q \gtrsim 0.8$ ), the initial perturbation becomes stabilized by accretion of a small amount of material, whereas for small $q$ the instability causes rapid accretion of the torus. The intensity of the threaded magnetic field influences the process of stabilization or destruction of the torus because, within the framework of the adopted model, the magnetic pressure adds directly to the gas pressure (plasma parameter $\beta \gtrsim 1$ ).

The process of accretion is not perfectly monotonic, instead, there are changing phases of enhanced accretion rate and phases where the mass of torus remains almost constant. The overall gradual decrease of the torus mass is superposed with oscillations that can be seen by following the central density variations on the dynamical time-scale and the position of the center of the torus. The oscillation amplitude is sensitive to the initial perturbation, but the frequency is not, namely, a small change of the metric coefficients does not affect the oscillation frequency. The toroidal magnetic field plays a more important role in the early phases of the accretion process until the perturbed configuration finds a new equilibrium or disappears because of the runaway instability. If the oscillations become stabilized with time, no significant differences occur compared to the corresponding non-magnetized case, even when $\beta$ is near unity (equipartition) and the accreted fraction of the torus material is significant.

Further discussion can be found in a recent paper (Hamerský \& Karas 2013). 


\section{References}

Abramowicz M. A., Karas V., \& Lanza A. (1998), A 6 A 331, 1143

Hamerský J., \& Karas V. (2013), A\&A 555, id. A32

Karas V., Huré J.-M., \& Semerák O. (2004), Classical and Quantum Gravity, 21, R1

Korobkin O., Abdikamalov E. B., Schnetter E., et al.(2013), Phys. Rev. D 83, id. 043007

Rezzolla L., Zanotti O., \& Font J. A. (2003), A\&A 412, 603 